\title{
Signaling mechanisms for regulation of chemotaxis
}

\author{
Dianqing $\mathrm{WU}^{*}$ \\ Department of Genetics and Developmental Biology, University of Connecticut Health Center, Farmington, CT 06030, USA
}

\begin{abstract}
Chemotaxis is a fascinating biological process, through which a cell migrates along a shallow chemoattractant gradient that is less than 5\% difference between the anterior and posterior of the cell. Chemotaxis is composed of two independent, but interrelated processes-motility and directionality, both of which are regulated by extracellular stimuli, chemoattractants. In this mini-review, recent progresses in the understanding of the regulation of leukocyte chemotaxis by chemoattractant signaling are reviewed.
\end{abstract}

Keywords: chemotaxis, leukocyte, signaling, chemoattractant.

\section{INTRODUCTION}

Leukocyte chemotaxis is regulated by a number of chemoattractants include bacterial by-product fMLP, complement proteolytic fragment $\mathrm{C} 5 \mathrm{a}$, and the superfamily $(\sim 50)$ of small (8-10 kDa), inducible, secreted, proinflammatory cytokines called chemokines $[1,2]$. These chemoattractants act as immediate mediators of inflammatory responses by regulating leukocyte recruitment, infiltration, homing, and trafficking as well as their development and function [3]. While inflammation plays an important role in host defense, uncontrolled inflammatory reactions are responsible for initiation and progression of many human diseases, including atherosclerosis, ischemia-reperfusion injury, virus-induced myocarditis, rheumatoid arthritis, allergic reactions, psoriasis and other inflammatory skin conditions, and even tumorigenesis and tissue-targeted metastasis [4-7]. However, the lack of specific therapeutic agents has impeded effective treatment of these inflammatory conditions. Thus, a better understanding of the regulation of leukocyte chemotaxis by chemoattractants may provide novel therapeutic targets or strategies to treat these diseases.

\section{CHEMOATTRACTANT SIGNALING PATHWAYS}

Chemoattractants bind to their specific cell surface receptors and mainly activate the Gi proteins in leukocytes [8]. The Gi proteins belong to the family of

*Correspondence: Dianqing WU

Tel: +860-679-8188; Fax: +860-679-1024

E-mail: dwu@neuron.uchc.edu heterotrimeric $\mathrm{G}$ proteins, which consist of three subunits: $\alpha, \beta$, and $\gamma$. The a subunits contain GTPase activity. Ligand-bound receptors promote the exchange of GDP with GTP on the a subunits, and the GTP-bound forms of G $\alpha$ subunits are subsequently dissociated from the $\beta \gamma$ subunits. While the a subunits of the Gi proteins inhibit adenylyl cyclases, the $\mathrm{G} \beta \gamma$ subunits can regulate a number of effectors, including phospholipase (PLC) $\beta 2 / 3$, phosphatidylinositide 3 (PI3K) $\gamma$, ion channels, G proteincoupled receptor kinases, and some subtypes of adenylyl cyclases [9]. We recently added PAK1 to the list by demonstrating the interaction between G $\beta \gamma$ and PAK1, which is involved in chemoattractant-mediated activation of Cdc42 and PAK1 [10]. Chemoattractants can also couple to G16 [11] and probably G12/13 [12], which have been shown to activate small GTPase RhoA via a guanine nucleotide exchange factor (GEF) 115 [13-15]. In the past ten years, many of the chemoattractant signaling pathways were comprehensively characterizing using a combination of biochemical, molecular and cell biological, and transgenic approaches. These pathways are summarized in Fig. 1A.

\section{SIGNALING MECHANISMS IN THE REGULATION OF DIRECTIONALITY}

Chemotaxis is a fundamental biological process in which a cell migrates following the direction of a spatial cue. This spatial cue is provided in a form of a gradient of chemoattractants. Cells ranging from prokaryotic bacteria to eukaryotic mammalian cells possess, to varying degrees, an ability to interpret the gradient, but through diverse mechanisms. For a bacterial cell, it is mainly a trial-anderror process in which the cell tumbles around and senses 
the surrounding periodically. If it tumbles into a location that contains a higher concentration of a chemoattractant, it continues in that direction for a while longer. If it senses that it has moved to a location that contains a lower level of a chemoattractant, it will change direction. By this trialand-error approach, the bacterial cell would eventually move up the gradient. The cells of higher organisms develop more sophisticated machineries to sense and process the information conveyed by the chemical gradient. These machineries allow a cell to tell small differences in chemoattractant concentration between its two ends (usually only a few percent) and translate this small difference into a much greater intracellular biochemical gradient. Neutrophils and Dictyostelium cells are two fine model systems for studying chemotaxis, both of which can efficiently interpret and chemotax under a shallow gradient of chemoattractants to allow observing, recording and analyzing their migration quantitatively using videomicroscopy. These analyses provide the means to discern the roles of signaling mechanisms in the regulation of directionality from those in regulating motility.

\section{MECHANISM OF CELL POLARIZATION IN REGULATION OF CHEMOTAXIS}

For a neutrophil to move even in a random migration, the cell has to polarize by forming F-actin-rich lamellipodia at the direction of the migration, because continuous formation of F-actin at the leading edge drives cell locomotion. Chemoattractants augment and stabilize cell polarization by stimulating $\mathrm{F}$ actin polymerization particularly in the lamellipodia; thus, the cell becomes highly polarized (Fig. 1B). When a chemoattractant gradient is present, the cell can sense the gradient and align its polarity with the gradient to migrate directionally up the gradient. Therefore, cell polarization provides a link between the two basic components of chemotaxis-motility and directionality. One of the characteristics of a highly polarized cell is its elongated shape with a more blunted leading edge and narrower posterior, resulting from the formation of $F$ actin-rich lamellipodia at the leading edge and actomyosin structure at the posterior (Fig. 1B). In addition, in a polarized cell many intracellular proteins, including $\mathrm{F}$ actin and lipids show polarized distribution (Fig. 1B). Among all these, the polarized distribution of a phospholipid, $\mathrm{PIP}_{3}$, which was initially observed in Dictyostelium [16] and later in neutrophils [17] and other cells [18], appears to occur prior to other polarization events. PIP3 is converted from $\mathrm{PIP}_{2}$ by PI3K [19]. Some of the Pleckstrin homology (PH) domains specifically recognize $\mathrm{PIP}_{3}$, thus leading to the translocation of the $\mathrm{PH}$ domain-containing proteins to the plasma membrane. Such translocation often leads to the regulation of the activity of these proteins that include many
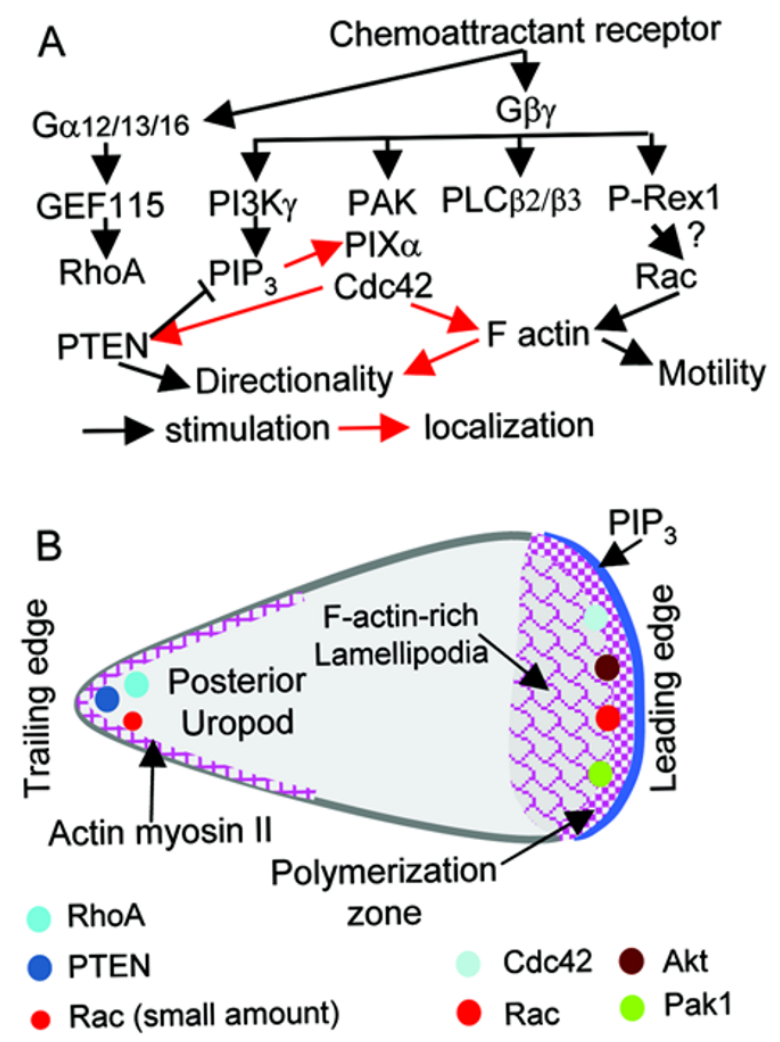

Fig. 1 Chemoattractant signaling pathways and neutrophil cell polarization. (A) Summary of chemoattractant signaling pathways and their actions in leukocyte chemotaxis. (B) Highly motile neutrophils develop high polarity upon stimulation. Only some of the proteins that show polarized distribution are shown.

protein kinases, GEFs, and structural proteins [19-22]. Chemoattractants have been shown to stimulate $\mathrm{PIP}_{3}$ production in leukocytes, presumably by activating a PI3K. The identification of PI3K $\gamma$, a G protein-regulated PI3K isoform [23, 24], led to the hypothesis that chemoattractants may stimulate $\mathrm{PIP}_{3}$ production via PI3K $\gamma$. To test the hypothesis and determine the significance of $\mathrm{PIP}_{3}$ in chemoattractant-induced chemotaxis, we and others generated PI3K $\gamma$-null mice [25-27]. PI3K $\gamma$-deficiency completely abrogated chemoattractant-induced $\mathrm{PIP}_{3}$ production in neutrophils, thus demonstrating that $\mathrm{PI} 3 \mathrm{~K} \gamma$ is required for chemoattractant-induced $\mathrm{PIP}_{3}$ production in mouse neutrophils. In addition, PI3K $\gamma$-deficiency resulted in impaired neutrophil and macrophage chemotaxis in response to a number of chemoattractants [25-27]. To better understand the mechanism by which $\mathrm{PI} 3 \mathrm{~K} \gamma$ regulates chemotaxis, we went on examining migratory and polarization characteristics of PI3K $\gamma$-deficient neutrophils in a shallow chemoattractant gradient. This study revealed that PI3K $\gamma$ or $\mathrm{PIP}_{3}$, although not required for neutrophils to polarize, is required for the cells to align their polarity with 
the chemoattractant gradient [28]. In other words, $\mathrm{PIP}_{3}$ is required for the cells to sense and interpret the spatial cue provided by the gradient and to orientate their polarity in accordance to the cue. Because the location of $\mathrm{F}$ actin or lamellipodia marks the direction of cell migration, the failure of preferential localization of $\mathrm{F}$ actin in accordance to the chemoattractant gradient explains the loss of directionality for PI3K $\gamma$-deficient cells. The study of PI3K-null Dictyostelium cells also yielded similar results [29]. More recently, we have gained further insights into how the $\mathrm{PIP}_{3}$ polarization regulates the orientation of cell polarity-by localizing the formation of $\mathrm{F}$ actin-rich lamellipodia at the side of the cell facing the source of chemoattractants. PIP 3 does this by localizing the activation of Cdc42 [10].

$\mathrm{Cdc} 42$ is a member of the Rho family of small GTPases [30-33]. Cdc42 is a key regulator of cell polarity in many cell types $[34,35]$ and directionality in macrophages [36]. Although it has been shown to activated by chemoattractants in leukocytes, the mechanism by which it is regulated by heterotrimeric $\mathrm{G}$ proteins remained elusive. We recently found that $G \beta \gamma$ could directly interact with PAK1 and activate PAK1 in the presence of Cdc42 and a small GTPase exchange factor PIX $\alpha$. PIX $\alpha$ is constitutively associated with PAK1 and can activate both Cdc42 and Rac in in vitro and overexpression assays [37, 38]. In addition, we observed that $\mathrm{G} \beta \gamma$ activated $\mathrm{Cdc} 42$ in the presence of PIX $\alpha$ and PAK1. Putting all these results together, we hypothesized that $\mathrm{G} \beta \gamma$ may activate $\mathrm{Cdc} 42$ by recruiting the PAK-PIX $\alpha$ complex to the plasma membranes, where PIX $\alpha$ activates $\mathrm{Cdc} 42$. Activated Cdc42 in turn activates its downstream effectors that include PAK1. This hypothesis was validated by the observation that chemoattractant-induced activation of $\mathrm{Cdc} 42$ required both PAK1 and PIX $\alpha$ in leukocytes as siRNAmediated suppression of PAK expression or inactivation of PIX $\alpha$ by gene targeting abrogated $\mathrm{Cdc} 42$ activation [10]. The fact that neither PAK1 suppression or PIX $\alpha$ inactivation affected chemoattractant-induced Rac1 activation suggest that PIX $\alpha$ functions as a specific $\mathrm{Cdc} 42$ exchange factor even though PIX $\alpha$ can activate both Rac1 and $\mathrm{Cdc} 42$ in in vitro or overexpression assay. In addition, our findings indicate that PAK1 is not only one of the effectors for $\mathrm{Cdc} 42$, but also functions as a scaffold protein that is required for Cdc 42 activation. A recent study using in vitro reconstitution assay has provided a mechanistic basis for the specific regulation of $\mathrm{Cdc} 42$ by PIX $\alpha$ upon chemoattractant stimulation. This study showed that G $\beta \gamma$, via interacting with PAK, activates the nucleotide exchange activity of PIX $\alpha$ toward Cdc42 by stimulating the dissociation of the PIX $\alpha$ dimer. PIX $\alpha$, when it is in the dimer form, shows little activity on Cdc42 [39].

It is interesting to note that this chemoattractant and
G $\beta \gamma$-mediated activation of Cdc 42 shares a close resemblance with a mechanism by which pheromone activates $\mathrm{Cdc} 42$ in yeast. In the yeast mechanism, a scaffold protein called Far $1 p$ bridges G $\beta \gamma$ to the guanine nucleotide exchange factor Cdc24 [40-42]. Mammalian homolog of Far1p has not been found; thus, we suspect that PAK1 may be the functional equivalent of Farlp. Consistent with the role of yeast $\mathrm{Cdc} 42$ in regulating cell polarity, the mammalian Cdc42 pathway also plays an important role in regulation of directionality during chemotaxis, i.e., aligning cell polarity with the chemoattractant gradient. Neutrophils that lack PIX $\alpha$ could no longer migrate following the gradient. Instead, these cells, like the PI3K $\gamma$-nullcells, wandered around with little sense of direction in a chemoattractant gradient [10]. As occurred to PI3K $\gamma$-null neutrophils, cells lacking PIX $\alpha$ also failed to localize F actin formation in accordance to the chemoattractant gradient despite that PIX $\alpha$-deficiency did not affect the formation of $F$ actin in response to chemoattractants [10].

\section{Role of PI3K $\gamma$-linked pathway and the PIX $\alpha$-Cdc42 pathway in directionality}

Because both PI3K $\gamma$-linked pathway and the PIX $\alpha$ $\mathrm{Cdc} 42$ pathway regulate directionality by regulating the localization of $\mathrm{F}$ actin, the relationship between these two signaling pathways was investigated. It seems that the PI3K $\gamma$ pathway may acts upstream of PIX $\alpha-\mathrm{Cdc} 42$ pathway, as $\mathrm{PI} 3 \mathrm{~K} \gamma$ is required for directional localization of active $\mathrm{Cdc} 42$. However, active $\mathrm{Cdc} 42$ and $\mathrm{F}$ actin are still co-localized in PI3K $\gamma$-null neutrophils despite the defective localization of active Cdc42. These observations indicate that $\mathrm{G} \beta \gamma$-mediated activation of PI $3 \mathrm{~K} \gamma$ determines the localization of active $\mathrm{Cdc} 42$, probably by regulating the localization of PIX $\alpha$ through its PH domain, and that active $\mathrm{Cdc} 42$ in turn determines where $\mathrm{F}$ actin is formed. Putting all these into the context of a cell, the polarization distribution of $\mathrm{PIP}_{3}$, whose production requires $\mathrm{PI} 3 \mathrm{~K} \gamma$, leads to polarized activation of $\mathrm{Cdc} 42$ and eventually to polarized distribution of $\mathrm{F}$ actin.

Knowing that the $\mathrm{PIP}_{3}$ gradient determines the localization of lamellipodia and thus the direction of cell locomotion, the ensuing questions are how the polarized distribution of $\mathrm{PIP}_{3}$ is established and orientated. Although we do not have a definitive answer to the question, one hypothesis suggests that this may be a result of amplification of a small receptor activation gradient corresponding to the ligand gradient through a cascade of positive feedbacks and negative regulations [43, 44]. Studies from Dictyostelium suggest that PTEN is one of the negative regulators that helps establishing and maintaining the $\mathrm{PIP}_{3}$ gradient $[45,46]$. PTEN is a protein and phospholipid phosphatase that can dephosphorylate PIP $_{3}[19,47-51]$. In chemotaxing 
Dictyostelium cells, PTEN was found to be translocated to the posterior membranes of the cells $[45,46]$. In chemotaxing neutrophils, we found that PTEN was also localized at the posterior and that this localization was dependent on PIX $\alpha$ [10]. Work is under the way for determining the mechanisms by which chemoattractants regulate PTEN via small GTPases. Because PTEN is a major tumor suppressor that is frequently mutated in a wide range of human tumor [52], elucidation of its regulation mechanisms, which still remain unclear, is clearly of great importance not only for better understanding chemotaxis, but also for its role in tumor suppression.

While $\mathrm{PIP}_{3}$ and $\mathrm{Cdc} 42$ localize $\mathrm{F}$ actin formation, they are not required for $\mathrm{F}$ actin formation because chemoattractants could still stimulate $\mathrm{F}$ actin formation in PI3K $\gamma$ or PIX $\alpha$-deficient neutrophils [10, 26, 53]. Thus, the next important question is how the actin polymerization is regulated by chemoattractants. Work using Rac-deficient neutrophils [54] and Rac dominant-negative mutants [55] has demonstrated the requirement of Rac for F-actin formation. What remains unresolved is how Rac is regulated by chemoattractants. A few years back, P-Rex 1 was identified as a G $\beta \gamma$ and PIP $_{3}$-regulated GEF that could activate Rac in in vitro and overexpression assays [56]. P-Rex 1 has since been believed to be the primary Rac activator in leukocytes, particularly in neutrophils. It is difficult to unambiguously determine the specificity of GEFs relying solely upon in vitro and overexpression assays because these GEF molecules, particularly their catalytic domains, share close amino acid sequence homology. Our study of PIX $\alpha$ provides an excellent example; PIX $\alpha$ was shown to activate both Rac and Cdc42 in vitro [37], but it only activates Cdc42 in neutrophils [10]. Thus, to unambiguously determine if P-Rex 1 is the major Rac regulator in vivo, study of P-Rex1-null mice will be required.

Chemotaxis is a complex biological process. What we have learned now may be only the tip of an iceberg. There are still many important questions that remain unanswered. In addition, chemotaxis is a process that is not only used by leukocytes. Neuronal and embryonic cells migrate during development. During angiogenesis, endothelial cells undergo chemotaxis to form blood vessels, while epithelial cells and fibroblasts chemotax during wound healing. Chemotaxis also has an important role in tumorigenesis, particularly tissue-targeted metastasis. Many cancer cells such as breast cancer cells are known to preferentially metastasize into certain tissues and organs. This preference has now been correlated with the production of chemoattractants by the target tissues and organs and upregulation of chemoattractant receptors in the cancer cells. Although findings on the regulation of chemotaxis are often from studies using neutrophils and Dictyostelium cells as model systems, many of the fundamental principles should be applied to other cell types, including those aforementioned.

\section{REFERENCES}

1 Zlotnik A, Yoshie O. Chemokines: a new classification system and their role in immunity. Immunity 2000; 12:121-7.

2 Baggiolini M, Dewald B, Moser B. Human chemokines: an update. Annu Rew Immunol 1997; 15:675-705.

3 Baggiolini M. Chemokines and leukocyte traffic. Nature 1998; 392:565-8.

4 Furie MB, Randolph GJ. Chemokines and Tissue Injury. Am J Pathol 1995; 146:1287-301.

5 Nathan C. Points of control in inflammation. Nature 2002; 420: $846-52$

6 Coussens LM, Werb Z. Inflammation and cancer. Nature 2002; 420:860-7.

7 Libby P. Inflammation in atherosclerosis. Nature 2002; 420: 868-74.

8 Murphy PM. The molecular biology of leukocyte chemoattractant receptors. Annu Rev Immunol 1994; 12:593-633.

9 Neer EJ. Heterotrimeric G proteins: organizers of transmembrane signals. Cell 1995; 80:249-57.

10 Li Z, Hannigan M., Mo Z, et al. Directional sensing requires G beta gamma-mediated PAK1 and PIX alpha-dependent activation of Cdc42. Cell 2003; 114:215-27.

11 Mao J, Yuan H, Xie W, Wu D. Specific involvement of G proteins in regulation of SRF by receptors. J Biol Chem 1998; 273: 27118-23.

$12 \mathrm{Xu}$ J, Wang F, Van Keymeulen A, et al. Divergent signals and cytoskeletal assemblies regulate self-organizing polarity in neutrophils. Cell 2003; 114:201-14.

13 Mao J, Yuan H, Xie W, Wu D. Specific involvement of GEF115 in activation of Rho and SRF by Ga13. Proc Natl Acad Sci USA 1998; 95:12973-6.

14 Kozasa T, Jiang XJ, Hart MJ, et al. P115 RhoGEF, a GTPase

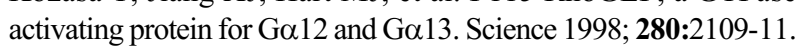

15 Hart MJ, Jiang XJ, Kozasa T, et al. Direct stimulation of the guanine nucleotide exchange activity of P115 RhoGEF by Ga13. Science 1998; 280:2112-4.

16 Parent CA, Blacklock BJ, Froehlich WM, Murphy DB, Devreotes PN. G protein signaling events are activated at the leading edge of chemotactic cells. Cell 1998; 95:81-91.

17 Servant G, Weiner OD, Herzmark P, et al. Polarization of chemoattractant receptor signaling during neutrophil chemotaxis. Science 2000; 287:1037-40.

18 Haugh JM, Codazzi F, Teruel M, Meyer T. Spatial sensing in fibroblasts mediated by 3' phosphoinositides. J Cell Biol 2000; 151:1269-80.

19 Vanhaesebroeck B, Leevers SJ, Ahmadi K, et al. Synthesis and function of 3-phosphorylated inositol lipids. Annu Rev Biochem 2001; 70:535-602.

20 Leevers SJ, Vanhaesebroeck B, Waterfield MD. Signalling through phosphoinositide 3-kinases: the lipids take centre stage. Curr Opin Cell Biol 1999; 11:219-25.

21 Irvine R. Inositol phospholipids: translocation, translocation, translocation. Curr Biol 1998; 8:R557-9.

22 Fruman DA, Rameh LE, Cantley LC. Phosphoinositide binding 
domains: embracing 3-phosphate. Cell 1999; 97:817-20.

23 Stephens LR, Eguinoa A, Erdjument-Bromage H, et al. The G beta-gamma sensitivity of a PI3K is dependent upon a tightly associated adaptor, p101. Cell 1997; 89:105-14.

24 Lopez-Ilasaca M, Crespo P, Pellici PG, Gutkind JS, Wetzker R. Linkage of $\mathrm{G}$ protein-coupled receptors to the MAPK signaling pathway through PI 3-kinase gamma. Science 1997; 275:394-7.

25 Li J, Yen C, Liaw D, et al. PTEN, a putative protein tyrosine phosphatase gene mutated in human brain, breast, and prostate cancer. Science 1997; 275:1943-7.

26 Hirsch E, Katanaev VL, Garland C, et al. Central role for G protein-coupled phosphoinositide 3-kinase gamma in inflammation. Science 2000; 287:1049-53.

27 Sasaki T, Irie-Sasaki J, Jones RG, et al. Function of PI3Kgamma in thymocyte development, T cell activation, and neutrophil migration. Science 2000; 287:1040-6.

28 Hannigan M, Zhan L, Li Z, et al. Neutrophils lacking phosphoinositide 3-kinase gamma show loss of directionality during N-formyl-Met-Leu-Phe-induced chemotaxis. Proc Natl Acad Sci U S A 2002; 99:3603-8.

29 Funamoto S, Milan K, Meili R, Firtel RA. Role of phosphatidylinositol 3' kinase and a downstream pleckstrin homology domain-containing protein in controlling chemotaxis in dictyostelium. J Cell Biol 2001; 153:795-810.

30 Bokoch GM. Regulation of cell function by Rho family GTPases. Immunol Res 2000; 21:139-48.

31 Hall A. Rho GTPases and the actin cytoskeleton. Science 1998; 279:509-14.

32 Etienne-Manneville S, Hall A. Rho GTPases in cell biology. Nature 2002; 420:629-35.

33 Fukata M, Nakagawa M, Kaibuchi K. Roles of Rho-family GTPases in cell polarisation and directional migration. Curr Opin Cell Biol 2003; 15:590-7.

34 Johnson DI. Cdc42: An essential Rho-type GTPase controlling eukaryotic cell polarity. Microbiol Mol Biol Rev 1999; 63:54-105.

35 Macara IG. Parsing the polarity code. Nat Rev Mol Cell Biol 2004; 5:220-31.

36 Allen WE, Zicha D, Ridley AJ, Jones GE. A role for Cdc42 in macrophage chemotaxis. J Cell Biol 1998; 141:1147-57.

37 Manser E, Loo TH, Koh CG, et al. PAK kinases are directly coupled to the PIX family of nucleotide exchange factors. Molecular Cell 1998; 1:183-92.

38 Daniels RH, Zenke FT, Bokoch GM. alphaPix stimulates p21activated kinase activity through exchange factor-dependent and -independent mechanisms. J Biol Chem 1999; 274:6047-50.

39 Feng Q, Baird D, Cerione RA. Novel regulatory mechanisms for the $\mathrm{Dbl}$ family guanine nucleotide exchange factor Cool-2/alphaPix. Embo J 2004; 23:3492-504.

40 Chant J. Cell polarity in yeast. Annu Rev Cell Dev Biol 1999; 15:365-91.

41 Nern A, Arkowitz RA. A Cdc24p-Far1p-Gbetagamma protein complex required for yeast orientation during mating. J Cell Biol 1999; 144:1187-202.

42 Nern A, Arkowitz RA. A GTP-exchange factor required for cell orientation. Nature 1998; 391:195-8.

43 Iijima M, Huang YE, Devreotes P. Temporal and spatial regulation of chemotaxis. Dev Cell 2002; 3:469-78.

44 Weiner OD. Regulation of cell polarity during eukaryotic chemotaxis: the chemotactic compass. Curr Opin Cell Biol 2002; 14:196-202.

45 Funamoto S, Meili R, Lee S, Parry L, Firtel RA. Spatial and temporal regulation of 3-phosphoinositides by PI 3-kinase and PTEN mediates chemotaxis. Cell 2002; 109:611-23.

46 Iijima M, Devreotes P. Tumor suppressor PTEN mediates sensing of chemoattractant gradients. Cell 2002; 109:599-610.

47 Di Cristofano A, Pandolfi PP. The multiple roles of PTEN in tumor suppression. Cell 2000; 100:387-90.

48 Leslie NR, Downes CP. PTEN: The down side of PI 3-kinase signalling. Cell Signal 2002; 14:285-95.

49 Wishart MJ, Dixon JE. PTEN and myotubularin phosphatases: from 3-phosphoinositide dephosphorylation to disease. Trends Cell Biol 2002; 12:579-85.

50 Sulis ML, Parsons R. PTEN: from pathology to biology. Trends Cell Biol 2003; 13:478-83.

51 Cantley LC, Neel BG. New insights into tumor suppression: PTEN suppresses tumor formation by restraining the phosphoinositide 3-kinase/AKT pathway. Proc Natl Acad Sci U S A 1999; 96:4240-5.

52 Eng C. PTEN: one gene, many syndromes. Hum Mutat 2003; 22:183-98

53 Li Z, Jiang H, Xie W, et al. Roles of PLC-beta2 and -beta3 and PI3Kgamma in chemoattractant-mediated signal transduction. Science 2000; 287:1046-9.

$54 \mathrm{Gu}$ Y, Filippi MD, Cancelas JA, et al. Hematopoietic cell regulation by Rac1 and Rac2 guanosine triphosphatases. Science 2003; 302:445-9.

55 Srinivasan S, Wang F, Glavas S, et al. Rac and Cdc42 play distinct roles in regulating $\mathrm{PI}(3,4,5) \mathrm{P} 3$ and polarity during neutrophil chemotaxis. J Cell Biol 2003; 160:375-85.

56 Welch HC, Coadwell WJ, Ellson CD, et al. P-Rex1, a PtdIns(3,4, 5)P3- and Gbetagamma-regulated guanine-nucleotide exchange factor for Rac. Cell 2002; 108:809-21. 